MPI-MIS-56/2000

hep-th/0009133

\title{
Hidden symmetries of supersymmetric $p$-form gauge theories
}

\author{
Friedemann Brandt \\ Max-Planck-Institute for Mathematics in the Sciences, Inselstraße 22-26, D-04103 Leipzig, Germany
}

Field theories with $p$-form gauge potentials can possess "hidden" symmetries leaving the field strengths invariant on-shell without being gauge symmetries on-shell. The relevance of such symmetries to supersymmetric models is discussed. They provide central charges of supersymmetry algebras, play a particular rôle in duality relations, and lead to peculiar interactions. A multiplet of $\mathrm{N}=2$ supersymmetry in four dimensions with two hidden central charges is presented.

PACS numbers: 11.30.Pb, 11.30.-j, 11.15.-q

Keywords: p-form gauge fields, supersymmetry, conservation laws, central charges, duality

This letter is devoted to a particular type of symmetries that field theories with $p$-form gauge potentials can have. These symmetries leave the field strengths invariant on-shell but are not gauge symmetries on-shell. For this reason they will be called "hidden" symmetries here. We shall first determine all such symmetries for Maxwelltype actions using results on the so-called characteristic cohomology of the field equations derived in 1 .

The remainder of the letter will focus on the relevance of hidden symmetries in the context of global supersymmetry. A particular aspect is that hidden symmetries can occur in the commutators of global supersymmetry transformations and then give "hidden central charges" of the supersymmetry algebra. This makes them play a particular rôle in dualities relating supersymmetry multiplets, among others. Another important aspect is the relevance of hidden symmetries to the construction of consistent supersymmetric interactions in theories with $p$-form gauge potentials.

A prominent supersymmetry multiplet with a hidden central charge symmetry is the vector-tensor (VT) multiplet of $N=2$ supersymmetry in four-dimensional spacetime [2] (see also [3]). Another example, a vector-tensortensor (VTT) multiplet with two hidden central charge symmetries, will be presented below.

The VTT multiplet illustrates also a related feature that will be discussed here and has been already observed in [4] when analyzing the $N=2$ double tensor multiplet: the on-shell commutator of two supersymmetry transformations may contain gauge transformations which involve explicitly the spacetime coordinates $x^{\mu}$, even when the supersymmetry transformations themselves do not depend explicitly on the $x^{\mu}$.

\section{CHARACTERISTIC COHOMOLOGY}

The hidden symmetries to be discussed are closely related to the so-called characteristic cohomology of the field equations which may also be called the "cohomology of the exterior derivative $d=d x^{\mu} \partial_{\mu}$ on-shell". We shall only sketch the basic concept here. The precise definition is made in so-called jet spaces whose coordinates are the spacetime coordinates and the fields and their derivatives, see e.g. [5] and references therein.

The cocycles of the characteristic cohomology are local $p$-forms $\omega_{p}$ (i.e., differential forms on some finite dimensional jet space) which are $d$-closed on-shell. This is denoted

$$
d \omega_{p} \approx 0,
$$

where $\approx$ denotes equality on-shell. In a Lagrangean field theory the field equations read $\hat{\partial} L / \hat{\partial} \phi^{i}=0$ where $\hat{\partial} L / \hat{\partial} \phi^{i}$ are the Euler-Lagrange derivatives of the Lagrangian with respect to the fields. By definition, equality on-shell is then equality modulo a combination of these EulerLagrange derivatives and derivatives thereof,

$$
X \approx Y: \Leftrightarrow X-Y=\sum_{k \geq 0} P^{i \mu_{1} \ldots \mu_{k}} \partial_{\mu_{1}} \ldots \partial_{\mu_{k}} \frac{\hat{\partial} L}{\hat{\partial} \phi^{i}},
$$

where $X, Y$ and the $P^{i \mu_{1} \ldots \mu_{k}}$ are local forms of the fields and the range of the summation index $k$ is finite.

A cocycle of the characteristic cohomology is called trivial (a coboundary) if it is $d$-exact on-shell,

$$
\omega_{p} \approx d \omega_{p-1} \text {. }
$$

The cocycles with form-degree $p=n-1$ (in $n$ dimensional spacetime) are conserved currents written as differential forms; the representatives with lower non-zero form-degree are sometimes called "higher order conservation laws". One can prove on fairly general assumptions, that the characteristic cohomology is locally trivial at all non-zero form-degrees $p<n-1$ for theories without nontrivial gauge symmetry. However, in gauge theories it may be nontrivial also at lower form-degrees. The lowest possible non-zero form-degree at which it can be nontrivial is then related to the reducibility order of the gauge symmetry [6]. 


\section{HIDDEN SYMMETRIES}

Let us first discuss purely bosonic actions of the Maxwell type,

$$
\begin{aligned}
S & =\frac{1}{2} \int d^{n} x \sum_{a} \frac{(-)^{p_{a}}}{\left(p_{a}+1\right) !} \sqrt{g} F_{\mu_{0} \ldots \mu_{p_{a}}}^{a} F^{a \mu_{0} \ldots \mu_{p_{a}}} \\
& =\frac{1}{2} \int \sum_{a}(-)^{n p_{a}} d A^{a} \wedge \star d A^{a} .
\end{aligned}
$$

$A^{a}$ are $p_{a}$-form gauge potentials with possibly different degrees $p_{a} \in\{1, \ldots, n-2\}, F_{\mu_{0} \ldots \mu_{p_{a}}}^{a}$ are the corresponding field strengths and $\star$ denotes Hodge dualization, using

$$
\begin{aligned}
A^{a} & =\frac{1}{p_{a} !} d x^{\mu_{1}} \wedge \ldots \wedge d x^{\mu_{p_{a}}} A_{\mu_{1} \ldots \mu_{p_{a}}}^{a} \\
d A^{a} & =\frac{1}{\left(p_{a}+1\right) !} d x^{\mu_{0}} \wedge \ldots \wedge d x^{\mu_{p_{a}}} F_{\mu_{0} \ldots \mu_{p_{a}}}^{a} .
\end{aligned}
$$

The spacetime metric $g_{\mu \nu}$ which occurs in the action is supposed to be a fixed background metric with Lorentzian signature $(+1,-1, \ldots,-1)$. The action is invariant under the gauge transformations

$$
\begin{aligned}
& \delta_{\text {gauge }}(\epsilon) A^{a}=d \epsilon^{a}, \\
& \epsilon^{a}=\frac{1}{\left(p_{a}-1\right) !} d x^{\mu_{1}} \wedge \ldots \wedge d x^{\mu_{p_{a}-1}} \epsilon_{\mu_{1} \ldots \mu_{p_{a}-1}}^{a}
\end{aligned}
$$

where $\epsilon_{\mu_{1} \ldots \mu_{p_{a}-1}}^{a}$ are arbitrary gauge parameter fields.

Let us now look for the hidden symmetries of the action. According to our definition, they are generated by transformations which vanish on-shell on the field strengths,

$$
\delta_{\text {hidden }} F_{\mu_{0} \ldots \mu_{p_{a}}}^{a} \approx 0 \Leftrightarrow d\left(\delta_{\text {hidden }} A^{a}\right) \approx 0 .
$$

Hence, $\delta_{\text {hidden }} A^{a}$ is $d$-closed on-shell. Furthermore it is not $d$-exact on-shell because otherwise $\delta_{\text {hidden }} A^{a}$ were a particular gauge transformation on-shell. Hence, $\delta_{\text {hidden }} A^{a}$ is a nontrivial cocycle of the characteristic cohomology of the field equations.

The relevant cohomology groups have been computed in a flat background in [1]. It can be checked that the computation in [1] goes through also in a general background. One obtains that the characteristic cohomology is represented (locally) at all form-degrees $0<p<n-1$ by the linearly independent exterior products of the Hodge dualized field strength forms $\star d A^{a}$ which can be built at the respective form-degree. Hence, $\delta_{\text {hidden }} A^{a}$ is a linear combination of such exterior products with formdegree $p_{a}$. In addition, $\delta_{\text {hidden }}$ must be a symmetry, i.e., it must leave the Lagrangian invariant modulo a total derivative. This is equivalent to the requirement that the Euler-Lagrange derivative of $\delta_{\text {hidden }} L$ with respect to each field $A_{\mu_{1} \ldots \mu_{p_{a}}}^{a}$ must vanish and gives

$$
\delta_{\text {hidden }} A^{a}=\sum c^{a a_{1} \ldots a_{r}}\left(\star d A^{a_{1}}\right) \wedge \ldots \wedge\left(\star d A^{a_{r}}\right)
$$

where the sum runs over all sets $\left\{a_{1}, \ldots, a_{r}: r=1,2, \ldots\right\}$ such that the form-degrees of the left and right hand sides in eq. (7) match,

$$
\sum_{i=1}^{r}\left(n-p_{a_{i}}-1\right)=p_{a}
$$

and the $c^{a a_{1} \ldots a_{r}}$ are constant coefficients with the following symmetry properties,

$$
\begin{aligned}
& c^{a_{0} \ldots a_{i} a_{i+1} \ldots a_{r}}=(-)^{\left(n-p_{a_{i}}-1\right)\left(n-p_{a_{i+1}}-1\right)} c^{a_{0} \ldots a_{i+1} a_{i} \ldots a_{r}} \\
& \left(\forall a_{i}, i=0, \ldots, r-1\right) .
\end{aligned}
$$

Eqs. (7) through (9) provide all hidden symmetries of an action (4). The corresponding Noether currents, written as local $(n-1)$-forms, are $j_{n-1}=\sum c^{a_{0} \ldots a_{r}}\left(\star d A^{a_{0}}\right) \wedge$ $\ldots \wedge\left(\star d A^{a_{r}}\right)$.

Suppose now that (4) is only one part of an action whose other part does not contain the fields $A^{a}$ but only additional ("matter") fields which do not bring in nontrivial gauge symmetries (i.e., the nontrivial gauge symmetries of the full action are still exhausted by (5)). Using the methods established in [6,1] one can then show on fairly general assumptions that the result on the characteristic cohomology described above remains valid, i.e., the matter fields give no contributions to the characteristic cohomology at form-degrees $<n-1$, at least locally (however, they may contribute at form-degree $n-1$ ). In particular, this holds when the action is quadratic in the matter fields. More generally, it holds for actions satisfying an appropriate "normality condition", see [6, 7, 5]. As an immediate consequence, the hidden symmetries of such actions are still exhausted by (7).

\section{LINEAR HIDDEN CENTRAL CHARGES}

We assume now that (4) is part of a globally supersymmetric action whose remaining part contains only matter fields and is quadratic in these fields. Accordingly, the supersymmetry transformations are assumed to be linear in the fields. One should have in mind here in particular models in a flat spacetime but the arguments are not restricted to that case. The linearity of the supersymmetry transformations implies that the hidden symmetries which can possibly occur in the commutators of supersymmetry transformations leave the matter fields invariant and act on the gauge fields according to

$$
\delta_{\text {hidden }}^{\text {linear }} A^{a}=\sum c^{a b} \star d A^{b}, \quad c^{a b}=(-)^{n p_{a}} c^{b a},
$$

where the sum runs over all values of $b$ such that $p_{a}=$ $n-1-p_{b}$. Furthermore, the transformations (10), and in fact all other transformations (\#) as well, commute on-shell with the supersymmetry transformations,

$$
\left[\delta_{\text {hidden }}, \delta_{\text {susy }}\right] \approx 0
$$


Obviously they also commute with the spacetime symmetries generated by Lie derivatives of the fields along Killing vector fields of the background metric.

These statements hold on simple and general grounds. Let us denote the commutator algebra of supersymmetry transformations on the field strengths and on the matter fields by $\left[\delta_{\text {susy }}, \delta_{\text {susy }}^{\prime}\right] \approx \delta_{\text {comm }}$ where $\delta_{\text {comm }}$ is some linear symmetry transformation. This gives $d\left(\left[\delta_{\text {susy }}, \delta_{\text {susy }}^{\prime}\right] A^{a}-\delta_{\text {comm }} A^{a}\right) \approx 0$. Using the results on the characteristic cohomology described above and the fact that $\left[\delta_{\text {susy }}, \delta_{\text {susy }}^{\prime}\right]-\delta_{\text {comm }}$ is a linear symmetry, one concludes $\left[\delta_{\text {susy }}, \delta_{\text {susy }}^{\prime}\right] A^{a}-\delta_{\text {comm }} A^{a} \approx \delta_{\text {hidden }}^{\text {linear }} A^{a}+d Y^{a}$ for some local forms $Y^{a}$ which are linear in the fields and may depend explicitly on the $x^{\mu}$ (see below). Hence, one has indeed $\left[\delta_{\text {susy }}, \delta_{\text {susy }}^{\prime}\right] \approx \delta_{\text {comm }}+\delta_{\text {hidden }}^{\text {linear }}+\delta_{\text {gauge }}(Y)$ with $\delta_{\text {hidden }}^{\text {linear as described above. }}$

(11) holds because linear supersymmetry transformations commute with the gauge transformations (5),

$$
\left[\delta_{\text {susy }}, \delta_{\text {gauge }}(\epsilon)\right]=0
$$

where the gauge parameter fields are inert to supersymmetry transformations, $\delta_{\text {susy }} \epsilon_{\mu_{1} \ldots \mu_{p_{a}-1}}^{a}=0$. Indeed, the commutator of any global symmetry transformation and a general gauge transformation (with arbitrary parameter fields inert to the global symmetry) either vanishes on-shell or generates a nontrivial gauge symmetry of the action on-shell. In our case $\left[\delta_{\text {susy }}, \delta_{\text {gauge }}(\epsilon)\right]$ is field independent when evaluated on any of the fields $\left(\delta_{\text {susy }}\right.$ is linear in the fields, while the gauge transformations (5) do not involve the fields). Hence, the field equations cannot appear in $\left[\delta_{\text {susy }}, \delta_{\text {gauge }}(\epsilon)\right]$ because they are linear in the fields. Furthermore $\left[\delta_{\text {susy }}, \delta_{\text {gauge }}(\epsilon)\right]$ is not a gauge transformation (5) as one has $\left[\delta_{\text {susy }}, \delta_{\text {gauge }}(\epsilon)\right] A^{a}=0$ $\left(\delta_{\text {susy }} \delta_{\text {gauge }}(\epsilon) A^{a}=0\right.$ owing to $\delta_{\text {susy }} \epsilon_{\mu_{1} \ldots \mu_{p_{a}-1}}^{a}=0$, and $\delta_{\text {gauge }}(\epsilon) \delta_{\text {susy }} A^{a}=0$ because $\delta_{\text {susy }} A^{a}$ is a linear combination of fermionic fields). This gives (12) and implies that a supersymmetry transformation of any field is gauge invariant and thus that the gauge fields $A_{\mu_{1} \ldots \mu_{p_{a}}}^{a}$ can enter the supersymmetry transformations only via the field strengths $F_{\mu_{0} \ldots \mu_{p_{a}}}^{a}$. This implies (11) because of $\delta_{\text {hidden }} F_{\mu_{0} \ldots \mu_{p_{a}}}^{a} \approx 0$.

Note that it only depends on the field content and the spacetime dimension whether or not there is a hidden symmetry (10): there must be at least two gauge potentials whose form-degrees add up to $(n-1)$ (these two gauge potentials may coincide if $n=4 k+1$ and $p_{a}=2 k$ ).

However, the fact that the hidden symmetries commute with the spacetime symmetries limits the possible situations in which they can be present in the commutators of supersymmetry transformations. For instance, Poincaré-invariant models in flat four-dimensional spacetime must have extended $(N \geq 2)$ supersymmetry in order that this can happen (see below).

\section{REMARK ON $X$-DEPENDENCE}

It should be stressed that all statements about the characteristic cohomology and hidden symmetries made above refer to the space of local forms and transformations which are allowed to depend explicitly on the spacetime coordinates $x^{\mu}$. This is relevant even in a flat background. Indeed, for an action (4), the characteristic cohomology at form-degrees $<n-1$ is bigger in the restricted space of $x$-independent local forms than in the space of all local forms. The additional representatives are exterior products of the $\star d A^{a}$ and at least one differential $d x^{\mu}[\mathbb{1}$. They give rise to symmetries of the action analogous to (7), but, in contrast to the latter, these symmetries are equal to gauge transformations on-shell and are thus trivial global symmetries according to modern terminology (cf. [5], section 6).

Of particular importance in the context of linear supersymmetry are the linear symmetries of this type because they can show up in the commutators of supersymmetry transformations (see [4] and the example below). They are given by

$$
\begin{aligned}
\delta_{\text {trivial }} A^{a} & =\sum c_{p}^{a b} \wedge\left(\star d A^{b}\right), \\
c_{p}^{a b} & =(-)^{\left(n-p_{a}-1\right)\left(n-p_{b}-1\right)} c_{p}^{b a}, \quad p>0
\end{aligned}
$$

where the sum runs over values of $b$ and $p$ such that $p_{a}=p+n-1-p_{b}$, and $c_{p}^{a b}$ are $p$-forms with constant coefficients,

$$
c_{p}^{a b}=\frac{1}{p !} c_{\mu_{1} \ldots \mu_{p}}^{a b} d x^{\mu_{1}} \wedge \ldots \wedge d x^{\mu_{p}}, \quad c_{\mu_{1} \ldots \mu_{p}}^{a b}=\text { constant }
$$

The $x$-dependent gauge transformations corresponding to (13) are easily found using $d x^{\mu}=d\left(x^{\mu}\right)$,

$$
\begin{aligned}
& \delta_{\text {trivial }} A^{a} \approx d X^{a} \Leftrightarrow \delta_{\text {trivial }} A^{a} \approx \delta_{\text {gauge }}(X) A^{a} \\
& X^{a}=\sum \frac{1}{p !} c_{\mu_{1} \ldots \mu_{p}}^{a b} x^{\mu_{1}} d x^{\mu_{2}} \wedge \ldots \wedge d x^{\mu_{p}} \wedge \star d A^{b} .
\end{aligned}
$$

\section{EXAMPLES IN 4 DIMENSIONS}

Examples in 4-dimensional spacetime involve only 1form or 2-form gauge potentials. Symmetries (10) then shift 1-form (2-form) gauge potentials by the Hodge dualized field strengths of 2 -form (1-form) gauge potentials. Hence, models with such symmetries must contain both at least one 1-form gauge potential and at least one 2form gauge potential.

Furthermore, Poincaré invariant models in flat 4dimensional spacetime must have extended $(N \geq 2)$ supersymmetry in order that the commutator of two supersymmetry transformations can contain a symmetry (10). This is seen when one writes the supersymmetry transformations as 


$$
\delta_{\text {susy }}=\sum_{i=1}^{N}\left(\xi^{\alpha i} D_{\alpha}^{i}+\bar{\xi}_{\dot{\alpha}}^{i} \bar{D}^{\dot{\alpha} i}\right)
$$

where $\xi^{\alpha i}$ are constant anticommuting Weyl-spinors, $\bar{\xi}^{\dot{\alpha} i}$ are their complex conjugates, and $D_{\alpha}^{i}$ and $\bar{D}_{\dot{\alpha}}^{i}$ generate the corresponding supersymmetry transformations of the fields (using conventions as in [8] for the Minkowski metric $\operatorname{diag}(1,-1,-1,-1))$. The commutator of two supersymmetry transformations involves the anticommutators $\left\{D_{\alpha}^{i}, \bar{D}_{\dot{\alpha}}^{j}\right\}$ and $\left\{D_{\alpha}^{i}, D_{\beta}^{j}\right\}$ (and the complex conjugates of the latter). $\left\{D_{\alpha}^{i}, \bar{D}_{\dot{\alpha}}^{j}\right\}$ contains no Lorentz-invariant piece and therefore it cannot contain a hidden symmetry (10). In contrast, $\left\{D_{\alpha}^{i}, D_{\beta}^{j}\right\}$ can contain $\varepsilon_{\alpha \beta} \delta_{\text {hidden }}^{i j}$ where $\delta_{\text {hidden }}^{i j}=-\delta_{\text {hidden }}^{j i}$ are hidden symmetries (10). The antisymmetry in $i, j$ requires $N \geq 2$.

The VT multiplet [2] meets these conditions. It contains one 1-form gauge potential, one 2-form gauge potential, one real scalar field and two Weyl fermions (one may add an auxiliary real scalar field). In that case one has $\left\{D_{\alpha}^{i}, D_{\beta}^{j}\right\} \approx \varepsilon_{\alpha \beta} \varepsilon^{i j} \delta_{\text {hidden }}$ with $\delta_{\text {hidden }}$ as in (10). Another example, the VTT-multiplet, will be given below.

Trivial symmetries (13) in four dimensions act nontrivially only on 2-form gauge potentials and involve constant 1 -forms $c_{1}^{a b}=-c_{1}^{b a}$. The antisymmetry in $a, b$ requires the presence of at least two 2 -form gauge potentials. As the $c_{1}^{a b}$ are 1-forms, these symmetries are not Lorentzscalars but Lorentz-vectors. Therefore they do not occur in $\left\{D_{\alpha}^{i}, D_{\beta}^{j}\right\}$ but in $\left\{D_{\alpha}^{i}, \bar{D}_{\dot{\alpha}}^{j}\right\}$ and this can happen already for $N=1$ supersymmetry.

A multiplet where a symmetry (13) occurs in the commutator of supersymmetry transformations is the $N=2$ double tensor multiplet [4]. Another example with $N=2$ supersymmetry is the VTT multiplet. An example with $N=1$ supersymmetry is the " $N=1$ double tensor multiplet" considered in [9]. It can be obtained by truncating the VTT multiplet, see below.

The VTT multiplet contains one 1-form gauge potential $A=d x^{\mu} A_{\mu}$, two 2 -form gauge potentials $B^{a}=$ $(1 / 2) d x^{\mu} \wedge d x^{\mu} B_{\mu \nu}^{a}(a=1,2)$ and two Weyl fermions $\psi_{\alpha}^{i}(i=1,2)$. It is convenient to combine the 2 -form gauge fields in complex fields,

$$
B=\frac{1}{2} d x^{\mu} \wedge d x^{\mu} B_{\mu \nu}, \quad B_{\mu \nu}=B_{\mu \nu}^{1}+\mathrm{i} B_{\mu \nu}^{2} .
$$

The Lagrangian for the free multiplet is

$$
L=-\frac{1}{4} F_{\mu \nu} F^{\mu \nu}-\frac{1}{2} H_{\mu} \bar{H}^{\mu}-2 \mathrm{i} \psi^{i} \partial \bar{\psi}^{i}
$$

where

$$
\begin{aligned}
F_{\mu \nu} & =\partial_{\mu} A_{\nu}-\partial_{\nu} A_{\mu}, \\
H^{\mu} & =\frac{1}{6} \varepsilon^{\mu \nu \rho \sigma} F_{\nu \rho \sigma}=\frac{1}{2} \varepsilon^{\mu \nu \rho \sigma} \partial_{\nu} B_{\rho \sigma} .
\end{aligned}
$$

The $\mathrm{N}=2$ supersymmetry transformations $D_{\alpha}^{i}$ to be discussed read

$$
\begin{gathered}
D_{\alpha}^{i} A_{\mu}=\varepsilon^{i j}\left(\sigma_{\mu} \bar{\psi}^{j}\right)_{\alpha} \\
D_{\alpha}^{i} B_{\mu \nu}=4\left(\sigma_{\mu \nu} \psi^{i}\right)_{\alpha}, D_{\alpha}^{i} \bar{B}_{\mu \nu}=0 \\
D_{\alpha}^{i} \psi_{\beta}^{j}=-\frac{i}{2} \varepsilon^{i j} \sigma_{\alpha \beta}^{\mu \nu} F_{\mu \nu}, D_{\alpha}^{i} \bar{\psi}_{\dot{\alpha}}^{j}=-\frac{1}{2} \delta^{i j} \bar{H}_{\alpha \dot{\alpha}} .
\end{gathered}
$$

The $\bar{D}_{\dot{\alpha}}^{i}$ are obtained by complex conjugation. The anticommutators $\left\{D_{\alpha}^{i}, \bar{D}_{\dot{\alpha}}^{j}\right\}$ read

$$
\begin{aligned}
\left\{D_{\alpha}^{i}, \bar{D}_{\dot{\alpha}}^{j}\right\} \psi_{\beta}^{k}= & -\mathrm{i} \delta^{i j} \partial_{\alpha \dot{\alpha}} \psi_{\beta}^{k} \\
& +\frac{\mathrm{i}}{2} \varepsilon_{\alpha \beta}\left(3 \delta^{j k} \delta^{i l}-\delta^{i j} \delta^{k l}\right) \partial_{\gamma \dot{\alpha}} \psi^{\gamma l} \\
\approx & -\mathrm{i} \delta^{i j} \partial_{\alpha \dot{\alpha}} \psi_{\beta}^{k} \\
\left\{D_{\alpha}^{i}, \bar{D}_{\dot{\alpha}}^{j}\right\} A_{\mu}= & -\mathrm{i} \delta^{i j} \sigma_{\alpha \dot{\alpha}}^{\nu} F_{\nu \mu} \\
\left\{D_{\alpha}^{i}, \bar{D}_{\dot{\alpha}}^{j}\right\} B_{\mu \nu}= & -\mathrm{i} \delta^{i j} \sigma_{\alpha \dot{\alpha}}^{\rho} F_{\rho \mu \nu} \\
& +\delta^{i j}\left(H_{\nu} \sigma_{\mu}-H_{\mu} \sigma_{\nu}\right)_{\alpha \dot{\alpha}} .
\end{aligned}
$$

The terms $H_{\nu} \sigma_{\mu}-H_{\mu} \sigma_{\nu}$ in $\left\{D_{\alpha}^{i}, \bar{D}_{\dot{\alpha}}^{j}\right\} B_{\mu \nu}$ make up a symmetry (13) and are thus $x$-dependent gauge transformations of $B_{\mu \nu}$ and $B_{\mu \nu}^{2}$ on-shell (see below). $F_{\nu \mu}$ and $F_{\rho \mu \nu}$ are modulo particular gauge transformations of $A_{\mu}$ and $B_{\mu \nu}$ equal to $\partial_{\nu} A_{\mu}$ and $\partial_{\rho} B_{\mu \nu}$ respectively. Hence, $\left\{D_{\alpha}^{i}, \bar{D}_{\dot{\alpha}}^{j}\right\}$ equals $-\mathrm{i} \delta^{i j} \partial_{\alpha \dot{\alpha}}$ plus gauge transformations on-shell.

The anticommutators $\left\{D_{\alpha}^{i}, D_{\beta}^{j}\right\}$ read

$$
\begin{aligned}
\left\{D_{\alpha}^{i}, D_{\beta}^{j}\right\} \psi_{\gamma}^{k} & =\mathrm{i} \varepsilon^{j k} \varepsilon^{i l} \varepsilon_{\alpha(\beta} \partial_{\gamma) \dot{\alpha}} \bar{\psi}^{\dot{\alpha} l}+(\alpha i \leftrightarrow \beta j) \approx 0 \\
\left\{D_{\alpha}^{i}, D_{\beta}^{j}\right\} \bar{\psi}_{\dot{\alpha} k} & =0 \\
\left\{D_{\alpha}^{i}, D_{\beta}^{j}\right\} A_{\mu} & =-\varepsilon^{i j} \varepsilon_{\alpha \beta} \bar{H}_{\mu} \\
\left\{D_{\alpha}^{i}, D_{\beta}^{j}\right\} B_{\mu \nu} & =-\varepsilon^{i j} \varepsilon_{\alpha \beta}\left(\varepsilon_{\mu \nu \rho \sigma} F^{\rho \sigma}+2 \mathrm{i} F_{\mu \nu}\right) \\
\left\{D_{\alpha}^{i}, D_{\beta}^{j}\right\} \bar{B}_{\mu \nu} & =0
\end{aligned}
$$

The second term in parantheses in $\left\{D_{\alpha}^{i}, D_{\beta}^{j}\right\} B_{\mu \nu}$ is a gauge transformation. Except for this gauge transformation, $\left\{D_{\alpha}^{i}, D_{\beta}^{j}\right\}$ is given by $-\varepsilon^{i j} \varepsilon_{\alpha \beta}\left(\delta_{\text {hidden }}^{1}-\mathrm{i} \delta_{\text {hidden }}^{2}\right)$ on-shell and contains two hidden symmetries:

$$
\begin{aligned}
& \delta_{\text {hidden }}^{a} A_{\mu}=H_{\mu}^{a}, \delta_{\text {hidden }}^{a} B_{\mu \nu}^{b}=\frac{1}{2} \delta^{a b} \varepsilon_{\mu \nu \rho \sigma} F^{\rho \sigma}, \\
& \delta_{\text {hidden }}^{a} \psi_{\alpha}^{i}=0 \quad(a, b=1,2) .
\end{aligned}
$$

Hence, the commutator of two supersymmetry transformations is given by the sum of a translation, two hidden symmetries (10) and gauge transformations on-shell,

$$
\begin{aligned}
{\left[\delta_{\text {susy }}, \delta_{\text {susy }}^{\prime}\right] \approx } & -a^{\mu} \partial_{\mu}+(a+\bar{a}) \delta_{\text {hidden }}^{1}-\mathrm{i}(a-\bar{a}) \delta_{\text {hidden }}^{2} \\
& +\delta_{\text {gauge }}\left(\epsilon_{\mu}=a^{\nu} B_{\nu \mu}-\mathrm{i} a_{\nu} x^{\nu} H_{\mu}+2 \mathrm{i} a A_{\mu}\right) \\
& +\delta_{\text {gauge }}\left(\epsilon=a^{\mu} A_{\mu}\right), \\
a^{\mu}= & \mathrm{i}\left(\xi^{i} \sigma^{\mu} \bar{\xi}^{\bar{j}^{\prime}}-\xi^{i \prime} \sigma^{\mu} \bar{\xi}^{i}\right), \quad a=\varepsilon^{i j} \xi^{i} \xi^{j \prime}
\end{aligned}
$$

where $\delta_{\text {gauge }}(\epsilon=\ldots)$ and $\delta_{\text {gauge }}\left(\epsilon_{\mu}=\ldots\right)$ are gauge transformations of $A_{\mu}$ and $B_{\mu \nu}$ with particular gauge parameter fields respectively $\left(\epsilon_{\mu}=\epsilon_{\mu}^{1}+\mathrm{i} \epsilon_{\mu}^{2}\right)$. Notice that the $x$-dependent gauge transformations in $\left[\delta_{\text {susy }}, \delta_{\text {susy }}^{\prime}\right]$ arise indeed from a symmetry (13) given by 


$$
\begin{aligned}
\delta_{\text {trivial }} B & =-\frac{\mathrm{i}}{2} d x^{\mu} a_{\mu} \wedge d x^{\nu} H_{\nu} \\
\Leftrightarrow \delta_{\text {trivial }} B^{a} & =\frac{1}{2} \varepsilon^{a b} d x^{\mu} a_{\mu} \wedge \star d B^{b} .
\end{aligned}
$$

If one sets $A_{\mu}=0, \psi_{\alpha}^{2}=0$ and drops $D_{\alpha}^{2}$, the above formulae provide the Lagrangian, the supersymmetry transformations and the commutator algebra of these transformations for the $N=1$ double tensor multiplet [9]. The symmetry (19) appears then in the $N=1$ commutators $\left\{D_{\alpha}^{1}, \bar{D}_{\dot{\alpha}}^{1}\right\}$.

\section{REMARK ON DUALITIES}

Assume that two linear supersymmetric models of the type considered above are related by a standard duality substituting an $(n-p-2)$-form gauge potential $\tilde{A}$ for a $p$-form gauge potential $A$ (including the case $p=0$ representing scalar fields). More precisely, the duality substitutes $\star d \tilde{A}$ for $d A$ both in the Lagrangian and in the supersymmetry transformations (with appropriate coefficients), and thus field equations for Bianchi identities (and vice versa). Hence, if an expression in the field strength components and their derivatives vanishes in the original model then its counterpart in the dual model vanishes at least on-shell.

As a consequence, the duality does not modify the on-shell supersymmetry algebra on the field strengths and matter fields (recall that the gauge fields occur in the supersymmetry transformations only via the field strengths). The only effect of the duality on the onshell algebra of supersymmetry transformations is thus a possible modification of the hidden symmetries and the gauge transformations which appear in the algebra. In particular, hidden symmetries (10) and/or trivial symmetries (13) may thus be present in the supersymmetry algebra after a duality transformation even when they were absent before.

For instance, the hidden central charge symmetry of the VT multiplet arises in this way by dualizing one of the two real scalar fields of an abelian $N=2$ vector multiplet. Similarly, the two hidden central charge symmetries (17) of the VTT multiplet arise by dualizing both scalar fields of an abelian $N=2$ vector multiplet. The latter duality gives also rise to the symmetry $(19)$ in the commutators of supersymmetry transformations. Analogous statements apply to the $N=2$ double tensor multiplet [4] and the $N=1$ double tensor multiplet [9] as these multiplets are dual to an $N=2$ hyper multiplet and an $N=1$ chiral multiplet respectively.

\section{CONSEQUENCES FOR INTERACTIONS}

Even though the previous discussions apply to linear models and supersymmetry transformations, they are also relevant to nonlinear supersymmetric extensions of such models. In particular they matter to the construction of interacting supersymmetric models from free ones. For instance, suppose that one looks for a nonlinear extension of a free supersymmetric model with a hidden symmetry occurring in the commutators of supersymmetry transformations. Then this hidden symmetry must have a counterpart in the algebra of supersymmetry transformations of the nonlinear model, i.e., the nonlinear model must have a symmetry of a similar type. In general this symmetry will be a nonlinear extension of the corresponding hidden symmetry of the free model. This can restrict the possible interactions quite severely. There are two possibilities to be distinguished:

(i) The hidden symmetry of the free model is extended to a nontrivial global symmetry of the interacting model. Examples have been constructed in 10 14 where the central charge symmetry of the free VT multiplet is extended to a nonlinear global symmetry.

(ii) The hidden symmetry of the free model is promoted to a gauge symmetry of the interacting model. This case is very interesting. In particular, the gauging of central charge symmetries is inevitable when one wants to couple a supersymmetry multiplet with such a symmetry to supergravity. Four-dimensional globally supersymmetric models with a gauged hidden central charge were constructed in 15 19], again for the VT multiplet. Supergravity models with VT multiplets were constructed in 20] (see also 21]).

In fact, the gauging of hidden symmetries is interesting in its own right, whether or not these symmetries occur in the commutator of supersymmetry transformations. It is a nontrivial matter already in the non-supersymmetric case, as was discussed for a particular example in [22] and in greater generality in [23]. Supersymmetry makes gauging of hidden symmetries even more involved because it requires to combine it with other interactions between $p$-form gauge fields and matter fields (in some cases it may even be impossible to gauge a hidden symmetry in a supersymmetric way).

The gauging of hidden symmetries may be combined with other interactions peculiar to $p$-form gauge potentials [24], such as Freedman-Townsend interactions 25,26] or couplings to Chern-Simons-forms. A large class of such supersymmetric models in four dimensions was constructed in 27] (see also [28]). Among others it was found there that gauged hidden symmetries in general do not commute with the supersymmetry transformations on-shell, in contrast to their counterparts in free models (cf. Eq. (11)). Analogous models in dimensions $n>4$ might be interesting especially in the string theory context but it seems that no such models have been constructed yet. 
[1] M. Henneaux, B. Knaepen and C. Schomblond, Commun. Math. Phys. 186 (1997) 137, hep-th/9606181.

[2] M.F. Sohnius, K.S. Stelle and P.C. West, Phys. Lett. B 92 (1980) 123.

[3] B. de Wit, V. Kaplunovsky, J. Louis and D. Lüst, Nucl. Phys. B 451 (1995) 53, hep-th/9504006.

[4] F. Brandt, New $\mathrm{N}=2$ supersymmetric gauge theories: the double tensor multiplet and its interactions, Nucl. Phys. B (to appear), hep-th/0005086.

[5] G. Barnich, F. Brandt and M. Henneaux, Local BRST cohomology in gauge theories, Phys. Rept. (to appear), hep-th/0002245.

[6] G. Barnich, F. Brandt and M. Henneaux, Commun. Math. Phys. 174 (1995) 57, hep-th/9405109.

[7] G. Barnich, F. Brandt and M. Henneaux, Commun. Math. Phys. 174 (1995) 93, hep-th/9405194.

[8] J. Wess and J. Bagger, Supersymmetry and supergravity, Princeton Series in Physics (Princeton Univ. Press, Princeton 1992).

[9] P.K. Townsend and P. van Nieuwenhuizen, Phys. Lett. B 109 (1982) 179.

[10] R. Grimm, M. Hasler and C. Herrmann, Int .J. Mod. Phys. A 13 (1998) 1805, hep-th/9706108.

[11] N. Dragon, S. Kuzenko and U. Theis, Eur. Phys. J. C 4 (1998) 717, hep-th/9706169.

[12] I. Buchbinder, A. Hindawi and B.A. Ovrut, Phys. Lett. B 413 (1997) 79, hep-th/9706216.

[13] N. Dragon and S. Kuzenko, Phys. Lett. B 420 (1998) 64, hep-th/9709088.

[14] E. Ivanov and E. Sokatchev, Phys. Lett. B 429 (1998) 35, hep-th/9711038.

[15] P. Claus, B. de Wit, M. Faux, B. Kleijn, R. Siebelink and P. Termonia, Phys. Lett. B 373 (1996) 81, hepth/9512143.

[16] P. Claus, B. de Wit, M. Faux and P. Termonia, Nucl. Phys. B 491 (1997) 201, hep-th/9612203.

[17] N. Dragon and U. Theis, Phys. Lett. B 446 (1999) 314, hep-th/9805199 (an earlier version is hep-th/9711025).

[18] N. Dragon, E. Ivanov, S. Kuzenko, E. Sokatchev and U. Theis, Nucl. Phys. B 538 (1999) 411, hep-th/9805152.

[19] U. Theis, Phys. Lett. B 486 (2000) 443, hep-th/0005044.

[20] P. Claus, B. de Wit, M. Faux, B. Kleijn, R. Siebelink and P. Termonia, Nucl. Phys. B 512 (1998) 148, hepth/9710212.

[21] P. Claus, B. de Wit, M. Faux, B. Kleijn, R. Siebelink and P. Termonia, Fortsch. Phys. 47 (1999) 125, hepth/9801200.

[22] F. Brandt and N. Dragon, in: Theory of Elementary Particles (Wiley-VCH, Weinheim 1998), hep-th/9709021.

[23] F. Brandt, J. Simón and U. Theis, Class. Quant. Grav. 17 (2000) 1627, hep-th/9910177.

[24] M. Henneaux and B. Knaepen, Phys. Rev. D 56 (1997) 6076, hep-th/9706119.

[25] V.I. Ogievetsky and I.V. Polubarinov, Yad. Fiz. 4 (1966) 216, Sov. J. Nucl. Phys. 4 (1967) 156.

[26] D. Freedman and P.K. Townsend, Nucl. Phys. B 177 (1981) 282.

[27] F. Brandt and U. Theis, Nucl. Phys. B 550 (1999) 495, hep-th/9811180.

[28] F. Brandt and U. Theis, Fortsch. Phys. 48 (2000) 41, hep-th/9901064. 